BULLETIN Bulletin hispanique

HISPANIQUE Université Michel de Montaigne Bordeaux

$116-2$ | 2014

Référentialité/autoréférentialité dans le roman espagnol contemporain : bilan et perspectives

\title{
Référence et autoréférence dans les romans de José María Merino
}

le motif de l'île dans El centro del aire (1991), El heredero (2003) et El lugar sin culpa (2007)

\section{Natalie Noyaret}

\section{(2) OpenEdition}

\section{Journals}

Édition électronique

URL : http://journals.openedition.org/bulletinhispanique/3430

DOI : 10.4000/bulletinhispanique.3430

ISBN : 979-10-300-0156-3

ISSN : $1775-3821$

Éditeur

Presses universitaires de Bordeaux

Édition imprimée

Date de publication : 1 décembre 2014

Pagination : 611-624

ISBN : 978-2-86781-963-6

ISSN : 0007-4640

Référence électronique

Natalie Noyaret, «Référence et autoréférence dans les romans de José María Merino », Bulletin hispanique [En ligne], 116-2 | 2014, mis en ligne le 01 décembre 2017, consulté le 19 avril 2019. URL: http://journals.openedition.org/bulletinhispanique/3430; DOI : 10.4000/bulletinhispanique.3430 


\title{
Référence et autoréférence dans les romans de José María Merino : le motif de l'île dans El centro del aire (1991), El heredero (2003) et El lugar sin culpa (2007)
}

\author{
Natalie Noyaret \\ Université de Caen
}

S'il s'avère possible de trouver un référent extérieur à quelques-unes des nombreuses îles qui servent de support aux trois cuvres analysées, force est de reconnaître que l'auteur pratique une référentialité peu marquée, distanciée, comme absorbée par la fiction. Plus encore, à travers le motif de l'île, ces romans ne font rien d'autre que se désigner eux-mêmes, que parler d'eux-mêmes.

Mots-clés : romans Merino, île, référentialité distanciée, autoréférence, hyperlittéralité.

Si es posible encontrar un referente externo para algunas de las numerosas islas utilizadas como soporte en las tres obras analizadas, hay que admitir que el autor practica una referencialidad poco marcada, distanciada, como si la absorbiera la ficción. Más aún, mediante el motivo de la isla, estas novelas no hacen más que designarse a si mismas, hablar de si mismas.

Palabras claves: Novelas Merino, isla, referencialidad distanciada, autorreferencia, hiperliteralidad.

If it was really possible to find an external referent for some of the numerous islands used as support in the three works we analyze, one would have to admit that the author uses distant, not emphasized references, as if they had been absorbed by fiction. Furthermore, throughout the motive of the island, these novels do nothing more than designate themselves, talk about themselves.

Keywords: Merino novels, island, referential distance, auto referentiality, hyperliterality. 


\section{$\grave{A}$} dire vrai, je ne voyais guère de sens à me tourner vers un autre écrivain que José María Merino à l'heure de rendre hommage à celle qui, percevant déjà là une grande écriture, sut m’orienter vers lui et son œuvre après avoir accepté, en 1996, d'être ma directrice de thèse. De fait, Geneviève Champeau allait guider et suivre ma recherche pendant une bonne quinzaine d'années, et je ne saurai jamais assez dire combien je lui en suis reconnaissante. Reconnaissante - cela va sans dire - de la formation qu'elle m’a donnée, mais aussi, comme je viens de le signaler, de m'avoir orientée vers "l'écrivain de León ", ainsi qu’on désigne parfois - et à juste titre - José María Merino, pourtant né à La Corogne, en 1941. Dès lors que j'ai eu découvert cet auteur - en 1996, il avait déjà publié, après des débuts en poésie, trois recueils de nouvelles, trois livres d'aventures "pour la jeunesse ", et quatre romans, dont La orilla oscura qui lui valut le Premio de la Critica en 1985 -, je n'ai eu de cesse de m'intéresser à sa production ultérieure dans toute son ampleur et sa variété générique, trouvant un enrichissement personnel et un plaisir sans cesse renouvelés dans les thématiques abordées et les procédés utilisés, certains d'eux particulièrement novateurs. Pourquoi, par ailleurs, ne pas traiter de quelque(s) ouvrage(s) de cet écrivain dans le cadre de cet hommage et au regard de l'axe de réflexion qui le fédère puisque " la référence et l'autoréférence constituent deux tensions présentes dans toute œuvre et dont seule varie l'importance relative ", ainsi que le précise Geneviève Champeau dans l'introduction aux actes du colloque de novembre 1992 consacré à la question ${ }^{1}$ ? Mais encore me fallut-il percevoir, en me penchant plus particulièrement sur les romans de Merino, que l'articulation entre les deux concepts (référence et autoréférence) se joue dans plusieurs d'entre eux à travers le motif de l'île, pour que mon choix, concernant l'écrivain, le corpus, et le sujet même de mon étude, fût définitivement arrêté. Il faut dire que, dans le cadre de cette importance qu'il confère dans ses fictions à la fonction de l'espace au point de l'élever au rang de personnage, l'île s'avère être l'un des deux motifs spatiaux privilégiés par José María Merino (l'autre étant le gouffre). À l'occasion de l'élaboration d'un article consacré à son œuvre récente (celle écrite entre 2000 et 2010$)^{2}$, j'ai, en effet, pu constater un recours fréquent à l'image de l'île - ce qui ne saurait étonner, ne serait-ce qu'en raison de l'importance qu'il accorde aux mythes - et une utilisation métaphorique de celle-ci, que ce soit en matière de représentation du temps, de l'espace imaginaire ou onirique, ou encore du texte qui la porte. Il m'intéresse aujourd'hui de creuser la question de la référence et de l'autoréférence à travers trois romans de cet écrivain fondés sur le motif de l'île - El centro del aire (1991), El heredero (2003) et El lugar sin culpa $(2007)^{3}$ - ; plus précisément, de voir comment et dans quelle mesure

1. Geneviève Champeau (Textes réunis par), Référence et autoréférence dans le roman espagnol contemporain, Bordeaux, Maison des Pays Ibériques, 1994, p. 10.

2. Natalie Noyaret, "José María Merino ", dans Natalie Noyaret (ed.), La narrativa española de hoy (2000-2010). La imagen en el texto (I), Bern, Peter Lang, 2011, p. 75-101.

3. El centro del aire, Barcelona, Alfaguara Hispánica, première édition 1991; El heredero, Barcelona, Alfaguara, première édition 2003; El lugar sin culpa. Los espacios naturales, Barcelona, Alfaguara, première édition 2007. Éditions de référence pour nos citations. 
l'île condense dans chacun d'eux l'une et l'autre tensions (référentielle et autoréférentielle). De même qu'une légère évolution se laisse observer au fil des ans dans les sujets traités par José María Merino à travers ses œuvres sans qu'il délaisse pour autant ses "obsessions " de toujours (principalement : la mémoire, l'identité, le double, l'Amérique $)^{4}$, peut-être faut-il s'attendre à des infléchissements - qu'il conviendra alors d'interpréter - en matière de référence et d'autoréférence, au gré de ces trois ouvrages dont la publication s'échelonne sur près de vingt ans. Quoi qu'il en soit, il s'agira chaque fois, pour le moins, de discerner et soupeser le rapport que ces récits entretiennent avec le réel et d'observer comment ce lien est signifié ; aussi, et plus largement, de voir se dégager une conception de la création romanesque, de l'œuvre littéraire en soi, et peut-être même de la littérature. Il semble indispensable néanmoins, avant toute chose, de procéder à un repérage des manifestations de l'île dans ces textes, et ce, en essayant de respecter l'ordre de l'apparition des différentes déclinaisons du motif pour mettre bien en lumière l'engendrement des formes.

\section{LîlLE ET SES DÉCLINAISONS (DANS LES TROIS ROMANS)}

Dans le cadre des récits qui nous occupent, l'île peut apparaître sous l'aspect de cette réalité géographique que nous connaissons tous, mais aussi se profiler à travers un élément d'une autre nature (un objet, un lieu, un concept...) qui trouve en elle sa métaphore.

Dans El centro del aire, un roman composé de huit parties dont chacune se divise en trois chapitres voués à livrer tour à tour le point de vue des trois personnages principaux de l'histoire (Bernardo, Julio Lesmes, Magdalena), l'île s'introduit par le biais du deuxième d'entre eux, ou plutôt par le regard que celui-ci porte sur la réalité qui l'entoure. Depuis que ses lectures d'enfant l'ont conduit à s'identifier à Robinson Crusoë, Julio Lesmes, dont le nom rappelle d'ailleurs celui de Jules Verne, assimile en effet en permanence son contexte spatial à une île tout aussi inhospitalière que celle sur laquelle échoua le célèbre héros aventurier. Les comparaisons rendent alors manifeste le processus analogique par lequel le personnage transpose systématiquement au contexte maritime tout élément ou aspect de son univers spatial : ainsi une ville visitée pour la première fois est-elle perçue "como nuevos parajes silvestres de aquella isla desierta en que su vida venía transcurriendo" (p. 28), et un lever du jour particulièrement livide "como la luz de la última playa del mundo" (p. 171). Par ailleurs, Julio Lesmes s'avère également exercer son activité d'écrivain - il a entrepris un roman sur le Patio qui abrita ses jeux d'enfant - en mimant le comportement de Robinson employé à récupérer tout élément de rebut pouvant l'aider à survivre sur son île, ce en quoi on peut voir signifiée, à un autre niveau, la relation de l'auteur au réel. Telle est, par exemple, l'attitude de

4. Cf. José María Merino, "Reflexión sobre mi narrativa a la luz del Quijote ", dans Irene Andres-Suárez y Ana Casas (eds.), Cuadernos de Narrativa, Neuchâtel, 1996, 1, p. 87. 
Julio Lesmes venant de retrouver dans sa mémoire le nom d'un compagnon de jeu disparu :

Y subrayó el nombre con la convicción de que se trataba de una pieza valiosa, olvidada por el náufrago, que habia sido arrebatada un día por la violencia del mar hasta ser arrastrada lejos de la vista, pero que devuelta a la playa de nuevo era comprendida en toda su importancia y recuperada con regocijo. (p. 220-221)

Or, les souvenirs ne sauraient suffire à eux seuls à faire revivre le Patio, qui fut notamment le cadre des aventures de pirates que s'inventaient ces enfants, lesquels avaient même imaginé une île de corail avec un atoll dans un coin de leur univers de jeu :

[...] su evocación del Patio estaba rodeada de trozos casi irreconocibles, donde se mezclaban rostros, pedazos de objetos y latas abolladas. El mar que golpeaba en el lejano atolón habia dejado sobre la playa aquellos recuerdos inconexos y sanguinolentos y a él no le quedaba patrimonio alguno. Pero debia seguir rebuscando para acumular los elementos y construir el Patio escrito que mantendría a raya, durante un tiempo, el acecho implacable del olvido. (p. 93)

Surmontant sa désolante improductivité, Julio Lesmes parvient effectivement, dans un premier temps, non point à recréer le Patio de son souvenir - à savoir, un Patio animé par le groupe qu'ils formaient et que rejoignit la petite Heidi, une enfant venue d'ailleurs et à l'imagination débordante -, mais tout au moins à en produire une description étonnamment minutieuse et ordonnée, comme pour le mettre à l'abri des assauts du temps. Dans une alliance du "faire " et du "dire ", ces quelque six pages de description, logées comme dans un écrin à l'ouverture du chapitre central de la troisième partie et séparées de la suite du texte par un blanc typographique, donnent à voir presque graphiquement l'île métaphorique qu'elles évoquent. Ce texte enclavé présente, en effet, le Patio comme un lieu à la fois maritime et fortifié, comportant à son tour une petite île dont le nom - Anguila - signale à la fois sa localisation dans l'espace (elle est située dans un coin du Patio) et sa nature fuyante et presque inaccessible (elle est pourvue de falaises abruptes et se recouvre en hiver d'un givre bleuté qui la rend presque invisible). On en découvrira sans doute d'autres aspects plus avant, mais ce qu'il importe ici de préciser encore, c'est que cet endroit presque parfait n'est qu'une préfiguration de celui - absolument parfait - que les personnages parviendront à entrevoir au terme du récit, au cours d'un voyage très bref effectué ensemble dans une île des Baléares. Imaginaire ou pas - on y reviendra -, toujours est-il que ce séjour sur l'île, relaté dans les deux dernières parties, permet non seulement au trio de rencontrer une femme et son enfant qui rappellent toutes deux fortement la Heidi disparue, mais aussi de pénétrer dans leur maison pour y découvrir la présence d'un Patio presque identique à celui de leur enfance. Seule Magdalena, la plus rationnelle des trois, osera s'y aventurer, pour y recevoir alors la vision hallucinante d'un groupe de jeunes pirates ayant ménagé une île dans leur terrain de jeu, et qui lui rappellent étrangement les enfants qu'ils furent... 
Les principes de mise en miroir et d'emboîtement d'îles les unes dans les autres sont également à l'œuvre dans El heredero, comme il transparaît déjà dans la division du roman en deux parties respectivement intitulées "Isclacerta" et "True Island". Deux titres qui correspondent, en réalité, aux noms de deux maisons, chacune d'elle assimilée, comme on l'aura compris, à une île, s'agissant pour la première d'une demeure de la région de León dans laquelle se déroule la première partie du roman, Pablo Tomás (le protagoniste-narrateur) s'étant rendu au chevet de sa grand-mère maternelle sur le point de mourir ; et pour la seconde, de la maisonnette où ce même Pablo Tomás est venu s'établir et fonder une famille, sur un îlot proche de la côte pacifique des États-Unis, endroit depuis lequel il écrit cette " chronique » qui est aussi une saga familiale. Mais c'est là n'avoir presque rien dit encore du vertigineux déploiement d'îles qui se produit dans ce roman, bon nombre d'entre elles n'étant certes, en réalité, que des maisons, lesquelles se donnent à voir chaque fois comme des répliques plus ou moins exactes d'une habitation qui serait à l'origine de toutes, et qu'il faut chercher, bien entendu, sur une île. Comme le suggère déjà le surnom - el Puertorriqueño - qui fut donné au bisaïeul de Pablo Tomás lorsqu'il revint des Amériques ainsi qu'on l'apprend dès le premier chapitre, la "première Isclacerta " se trouve en vérité sur l'île de Porto Rico, ayant été la villa que l'arrière grand-père - figure par excellence de l'indiano - occupa làbas et qu'il chercha à reproduire à l'identique dès lors qu'il revint sur sa terre d'origine. Une Isclacerta qui, isolée au milieu des bois, s'apparente à une île, ce que viennent rehausser encore les images maritimes qui participent à son évocation. Pablo Tomás ne manquera pas d'ailleurs d'observer la ressemblance entre les deux bâtisses lorsque, destiné, semble-t-il, à reproduire le parcours de cet ancêtre à qui il s’assimile sans doute de façon excessive, il se rendra à son tour sur l'île caribéenne dans la deuxième partie du roman. Et c'est même en termes de « reconnaissance» d'un lieu pourtant jamais visité par lui jusqu'alors qu'il remémore son arrivée à Porto Rico où il ne s'établira pourtant pas, en conformité avec les variations que Merino introduit toujours dans la répétition du « même " qu'il cultive à l'envi dans ce roman, cherchant ainsi à susciter une réflexion sur l'identité vue comme un destin ${ }^{5}$. Mais, pour nous en tenir au processus de multiplication des îles ("réelles » et métaphoriques), on observera qu'il induit une dialectique entre le contenu et le contenant, entre la grandeur et la petitesse, mais aussi entre la réalité et la fiction (les petites maisons étant parfois comparées à des jouets), tout ceci expliquant sans doute le penchant de Merino à introduire des miniatures dans ses récits. Preuve en est ici la présence d'une maison de poupée au centre même d'Isclacerta, disposée comme sur un autel au milieu de la chambre de la mourante ; un cadeau du Puertorriqueńo à sa première épouse, dont la grand-mère hérita ensuite, et qui, à la mort de cette dernière, sera réclamé par Pablo Tomás comme seul héritage, chacun de ces personnages, avec son vécu personnel, trouvant en cet objet matière à se

5. Selon ce qu'il explique dans "Secretos de El heredero ", El heredero, edición, introducción y notas de Fernando Valls, Madrid, Barcelona, Clásicos Castalia, 2011, p. 79. 
constituer tout un monde imaginaire propre à compenser les frustrations de la vie réelle. Véritable talisman chargé de vertus et de secrets (p. 93), la maison de poupée est, essentiellement, "un lugar mental en el centro del aparente desorden de todo" (p. 109), en quelque sorte, là encore, "le centre de l'air "... mais c'est déjà entrer dans l'autoréférentialité et définir le roman en lui-même. En revanche, ce sera toucher à la question de la référence que d'ajouter que, dans cette œuvre, l'île trouve encore sa déclinaison dans l'Ellis Island visitée par une amie de Pablo Tomás lors de son passage par New York, «la isla de Ellis » avec son musée de l'émigration, qui donne à voir des vies entières de familles en miniatures, ce à travers quoi l'auteur du roman pose, d'une autre manière, le problème - qui est celui de son personnage - de l'enracinement de l'homme en quelque endroit de la terre : «tu manía del arraigo y del desarraigo » (p. 173). Reste à ajouter que ce thème motive également le métarécit qui vient dialoguer par fragments avec l'histoire principale, à savoir un roman de gare relevant de la science-fiction, biais par lequel, sous le franquisme, le grand-père Alberto attaquait subtilement les tenants du régime au pouvoir en racontant l'invasion de la Terre par une espèce végétale à la recherche d'un autre astre que le sien, sur lequel se fixer et se reproduire. Et ces légumes extraterrestres aux allures militaires de s'exclamer, en arrivant sur la Terre Promise : «sklakert» (p. 27), dont les sonorités rappellent "Isclacerta ». Inutile de dire que d'autres endroits du monde font encore figure d'îles dans ce roman, toutes concourant à la quête de cette " Île Vraie ", la "Isla Verdadera " que Pablo Tomás dit enfin avoir trouvée, au terme de son récit, en cet îlot du Pacifique qui a alimenté des histoires de baleines, et qui est sans doute plus fictif que " réel ».

À l'inverse, dans El lugar sin culpa, il n'est qu'une île pour seul et unique lieu diégétique, mais une Île ô combien Vraie ("Certa»), ô combien essentielle, même si, au terme du récit, elle se révèle n'avoir été qu'un lieu imaginaire. Assurément, des trois récits dont on traite, celui-ci est-il celui qui procure le plus fortement un effet d'illusion référentielle, non seulement parce qu'il ne s'affiche pas aussi ostensiblement que les deux autres (surtout le premier, sur lequel semble peser encore l'expérimentalisme des années soixante-dix) comme un roman de métafiction, mais aussi en raison du traitement " réaliste " dont il est l'objet ${ }^{6}$, notamment dans sa composante spatiale. El lugar sin culpa est au nombre de ces œuvres qui invitent tout particulièrement à se demander non point " comment la littérature copie la réalité " mais bien plutôt comment elle "nous fait croire qu'elle copie la réalité ", selon la nuance introduite par Philippe Hamon dans "Un discours contraint " ${ }^{7}$. Mis d'emblée en présence de la doctora Gracia sur l'île presque déserte où elle est venue se retrouver, oublier une vie familiale décevante et ne vivre que dans l'instant présent,

6. Voir les déclarations de l'auteur dans Diego Marín A., "Ahora se consume mucha pseudoliteratura, filones comerciales ", Diario La Rioja, Cultura, 28/11/2006 [dernière consultation le 6 mai 2013]. Disponible sur :http://www.larioja.com/.../ahora-consume-muchapseudoliteratura_20061128.html

7. Philippe Hamon, "Un discours contraint ", dans R. Barthes et alii, Littérature et réalité, Paris, Seuil, 1982, p. 132. 
aspirant même à se métamorphoser en l'un de ces lézards qui sont sa plus proche compagnie, le lecteur de ce bref roman se laisse assurément prendre au mirage du réalisme recherché par l'auteur, non seulement à travers un recours au présent et à la troisième personne de narration censé offrir "la perspectiva de la experiencia real de una persona ${ }^{8}$ mais aussi par le biais d'une évocation minutieuse, proprement visuelle, de l'île 9 . Et pour cause : cette histoire n'aura été, très probablement - Merino ménage néanmoins un certain doute jusqu’à la fin -, qu'une échappée onirique de sa protagoniste sur une île dont elle contemple la photographie - qui motive d'ailleurs une belle ekphrasis - chaque fois qu'elle se retrouve dans la salle d'attente de son psychiatre. Or, ne peut-on voir là signifié que, pour autant qu'une réalité non verbale préexiste à la fiction, la relation directe entre mots et référents n'est qu'un leurre, qu'une "illusion ", pour reprendre le mot de Michael Riffaterre ${ }^{10}$. En cela, l'auteur de ces romans ne saurait nous contredire.

\section{Des ÎleEs, EN RÉFÉRENCE. MAIS QUeLLES ÎLES ?}

Tout comme il part toujours du réel quotidien pour écrire ses nouvelles fantastiques, José María Merino forge généralement le contexte spatial, et même spatio-temporel, de ses romans sur la base de sa propre expérience. Toutefois, comme il le précise lui-même dans ses écrits théoriques, dès lors que les lieux visités passent dans le champ de la fiction littéraire, ils perdent leur condition d'espaces de la réalité brute pour devenir des espaces de la réalité imaginaire : "dejan de pertenecer al fluir sin forma, cifra ni destino de lo que sucede, para incorporarse a la estructura ordenada de la ficción, donde pueden alcanzar esa coherencia poética que el arte y la literatura consiguen dar incluso a los más extremados productos de la locura ${ }^{11}$. Et l'écrivain d'expliquer par exemple, au sujet de El heredero, dans un texte qui accompagne la réédition de ce roman dernièrement effectuée par Fernando Valls pour les Clásicos Castalia, que certains " décors " de ce récit - Merino préfère parler de "escenarios" plutôt que de "espacios" - correspondent à des lieux de sa connaissance, visités lors de voyages (Porto Rico, Lisbonne, une université nord-américaine, etc.), tandis que d'autres, ainsi que la maison de poupée, sont en rapport avec des endroits qui ont toujours exercé sur lui une certaine fascination. À l'origine de la création d'Isclacerta, se trouverait, en l'occurrence, quelque maison isolée

8. José María Merino, dans Diego Marín A., "Ahora se consume... ", art. cit.

9. Voir à ce sujet mes commentaires dans Natalie Noyaret, "José María Merino ", art. cit., p. 89-90.

10. Michael Riffaterre, "Lillusion référentielle ", dans R. Barthes et alii, Littérature et réalité, op. cit., p. 92.

11. José María Merino, «El narrador narrado », texte prononcé lors du « Grand Séminaire sur l'œuvre de José María Merino ", Universidad de Neuchâtel, 14-16 mai 2001, dans José María Merino, Ficción continua, Barcelona, Seix Barral, 2004, p. 26. 
dans la mythique vallée de Babia ${ }^{12}$. De même, l'espace temporel de ce roman relève de la propre trajectoire vitale de l'auteur, de ce retour sur les $\mathrm{XX}^{\mathrm{e}}$ et $\mathrm{XIX}^{\mathrm{e}}$ siècles que le passage au troisième millénaire le conduisit à effectuer : une sorte de prise de conscience soudaine de la fin d'une époque, qui lui fit élaborer cette histoire d'une famille espagnole sur quatre générations, avec la dimension symbolique qui s'ensuit sur le plan de l'histoire collective. Pour en revenir aux îles, celles qui apparaissent dans El centro del aire et dans El lugar sin culpa lui furent, d'après son témoignage, inspirées par les îles Baléares, qu’il découvrit en naviguant à bord d'un voilier pendant plusieurs étés, des îles sur lesquelles il est intéressant d'observer qu'il posait toutefois un regard littéraire : "alli encontré lugares que parecían el escenario previo a la llegada de Robinsón ${ }^{13}$. Cela étant, c'est Formentera qui, à travers quelques détails ou quelque périphrase, se laisse deviner derrière l'île, jamais nommée, où se rendent les personnages de El centro del aire, tandis que celle de Cabrera, aujourd'hui Parc National, se laisse reconnaître dans l'espace protégé dans lequel se déroule El lugar sin culpa, un roman que l'auteur annonça d'ailleurs comme le premier volume d'un cycle consacré aux «Espacios naturales ». Là encore, l'identification du référent spatial est rendue possible, pour qui veut se livrer à cet exercice, par un ensemble d'éléments descriptifs ou culturels (historiques, en l'occurrence ${ }^{14}$ ) distribués ci et là au fil de la narration. Ainsi parlerai-je d'une référentialité en pointillés ou opaque à propos de ces romans, à moindre mesure en ce qui concerne El heredero, dont la portée historique obligeait sans doute à davantage de transparence: Porto Rico est tout au moins citée, ainsi que les principales villes de cette île. Cela étant, force est d'observer la tendance de José María Merino à cultiver l'imprécision en matière de référence à la réalité extra-textuelle, ce qu'il reconnaît et justifie en ces termes: "Generalmente he procurado huir de las referencias concretas en mis libros. Creo que cada novela plantea en esto un problema diferente, y que el acierto - o logro - no está en la posible familiaridad del lector sino en el poder de sugestión del autor. $"{ }^{15}$ Ainsi eut-il soin d'échapper, dans El lugar sin culpa, au "danger d'une certaine évidence naturaliste " en évitant les références spatiales et historiques ponctuelles, ce qui lui permit aussi de conférer une dimension mythique, une valeur atemporelle à son roman. Ceci me semble valoir aussi pour les deux autres ouvrages. Mais j'en reviendrai encore à El heredero pour observer que ce n'est que par la référence à Melville

12. Cf. José María Merino, «Secretos de El heredero », art. cit., p. 77-78.

13. Cf. José María Merino, dans « He querido contraponer la vida humana, racional, a la impasibilidad atemporal de la naturaleza », entrevista parcialmente editada el 26/04/2007 por el Dpto. prensa Alfaguara [dernière consultation le 6 mai 2013]. Disponible sur :

http : //www.alfaguara.com/.../dossier-prensa-lugar-sin-culpa...

14. Ainsi, par exemple, dans ces lignes : "El asunto de la foca muerta y del derrumbe del murete de las ruinas paleocristianas hace que el arqueólogo se olvide por una vez de recordar el lugar que atraviesan, el espacio donde al parecer se amontonaban los muertos, en los primeros años del siglo diecinueve, cuando la isla fue utilizada como campo de concentración para los soldados franceses prisioneros en una batalla memorable y deportados alli." (El lugar sin culpa, op.cit., p. 24).

15. José María Merino, dans Carlos Javier García, La invención del grupo leonés, Gijón, Júcar, 1994, p. 98. 
et Moby Dick apportée par le protagoniste-narrateur que l'on peut déduire que l'île sur laquelle il s'est finalement établi se trouve sur la côte du Massachusetts, en face de l'île de Nantucket. La référence rejoint donc l'autoréférence, si tant est qu'on veuille bien inclure dans celle-ci le fait que « la fiction se constitue dans un dialogue intertextuel et ne dit le monde qu'en référant à d'autres textes ", ainsi qu'il fut envisagé lors du colloque de novembre $1992^{16}$. Quoi qu'il en soit, je saisis l'occasion d'observer au passage l'importance des références littéraires dans les récits dont on traite, ce qui tient sans doute en partie au motif de l'île, si fécond en littérature, sans parler du poids que peut exercer une œuvre comme l'Odyssée, dans laquelle Merino voit « le modèle primordial des paysages et des aventures de la tradition romanesque $»^{17}$. Mais, pour en finir avec la tension référentielle dans ces textes et continuer d'approcher l'autre pôle dont on aura compris qu'il prévaut sur le précédent, je signalerai cet autre phénomène : à savoir qu'il se trouve aussi dans ces romans des éléments spatiaux qui, de prime abord, semblent relever de l'autoréférence, telle la présence d'un monument élevé en hommage à l'auteur du roman Hector Servadac sur l'île où se rendent les personnages de El centro del aire, ou encore la mention d'une obélisque érigée sur l'île de El lugar sin culpa, et qui n'en correspondent pas moins à des réalités existantes sur les îles de référence. On y verra l'un de ces « tours » que Merino joue parfois à son lecteur, allant même ici jusqu'à mettre en avant la nature fictive de ces éléments ("su figuración de que aquello parecía una realidad ficticia se hizo aún mayor", "El centro del aire ", p. 332). Mais force est de reconnaître qu'ils sont propres à fonctionner sur les deux plans (référentiel et autoréférentiel).

\section{Hyper-LitTÉRALITÉ ET MOTIF DE L'ÎlLE}

Indépendamment du fait que l'autoréférence revêt un caractère explicite dans El centro del aire où Merino théorise sur la littérature et son rapport au réel à travers la personne de Julio Lesmes dont il fait son porte-parole, ces romans sont sous-tendus par une tension autoréférentielle de nature plus indirecte, perceptible à travers certaines procédures particulièrement manifestes dans le traitement des îles. Quand bien même celles-ci auraient-elles un référent extérieur, ces textes ne font rien d'autre, en filant et déclinant ce motif dans lequel ils trouvent leur plus belle métaphore, que de se désigner eux-mêmes et de parler d'eux-mêmes. Soit une sorte de « court-circuit référentiel » qui relève de leur sens «hyper-littéral», pour reprendre un mot de Frédéric Bravo ${ }^{18}$.

16. Geneviève Champeau (Textes réunis par), Référence et autoréférence..., op. cit., p. 8.

17. José María Merino, «Los parajes de la ficción », texte prononcé lors du congrès "Visiones del paisaje ", Universidad de Córdoba, Priego de Córdoba, novembre 1997, reproduit dans José María Merino, Ficción continua, op. cit., p. 103.

18. Frédéric Bravo, "L'analyse littérale ", dans Nadine Ly (Textes réunis par), Littéralité 3. Limage dans le tapis, Bordeaux, Presses Universitaires de Bordeaux, Maison des Pays Ibériques, 1997, p. 52. 
Une première étape de la démonstration, anticipée par mon observation sur le poids des références littéraires dans ces textes, consistera à observer que ceux-ci, en leur qualité de "mondes possibles $»^{19}$, ne cessent en fait de dévoiler leur statut de constructions culturelles et/ou imaginaires par le biais d'indices propres à suggérer la nature fictionnelle et/ou fictive des îles dont il est question en leur sein. Indices d'intertextualité, certes, comme la référence implicite à l'héroïne de Johanna Spyri à travers la petite Heidi qui incarne l'invention enfantine dans El centro del aire (et ce n'est qu'un exemple parmi d'autres, dont quelques-uns relèveraient de l'intertextualité " restreinte $»^{20}$ ), mais aussi références cinématographiques ou picturales, telle celle apportée au moment où le protagoniste de El heredero découvre Porto Rico, tant et si bien qu'on en viendrait à se demander pour un peu si ce qu'il vit sur cette île (en l'occurrence, sa vision, dans la maison qui fut celle du Puertorriqueño, d'une vieille femme noire allongée sur un sofa, dans une sorte de dédoublement - "imagen espejeada $»^{21}$ - de la grand-mère espagnole) ne serait pas, tout simplement, la transposition d'un tableau de Leighton contemplé à son arrivée au Musée d'art de la ville de Ponce. Signes d'artificialité, de fausseté, renvois à la représentation artistique sont aussi de mise, dans les trois récits, pour signaler l'essence fictive des îles qu'ils renferment. Quant à la vocation qu'ont ces dernières de refléter le texte qui les porte, elle est signifiée, à l'endroit de leur évocation, par un recours intense au lexique de la brillance et de la réverbération, signal d'autoréférentialité s'il en est. Si l'on écoute en effet Frank Wagner s'appuyant lui-même sur Jean Ricardou, "la réflexion (spéculaire) [...] occasionne un retournement de la fonction représentative "traditionnelle" (telle que la conçoit et met en œuvre le récit réaliste) en vertu duquel "le récit se désigne mille fois lui-même" "22. D’où, par exemple, «la brillance métallique » de l'eau qui entoure l'île de El lugar sin culpa, une eau dont la couleur et la "singulière opacité " peuvent renvoyer au support photographique; ou encore la description de l'archipel sur lequel se rend le trio de El centro del aire, tel que Julio Lesmes l'aperçoit depuis l'avion :

19. Rappelons que l'application, dans les années 1980, à la fiction littéraire des théories philosophiques des "mondes possibles " (elles-mêmes issues de la sémantique de Kripke) fut le fait de théoriciens tels que David Lewis, Thomas Pavel ou encore Umberto Eco (ainsi qu'il apparaît notamment dans Lector in fabula. Le rôle du lecteur, Paris, Grasset, 1985, p. 167 en particulier).

20. En l'occurrence, l'image de la maison isolée du reste du monde telle que se présente Isclacerta dans El heredero était déjà apparue dans la nouvelle de José María Merino «El dibujo de la nieve " (Días imaginarios, Barcelona, Seix Barral, 2002, p. 231-236). D'ailleurs, il est à noter que, dans le roman, Isclacerta a été l'objet de dessins au crayon de la part de la grand-mère, précieusement conservés par Pablo Tomás.

21. Selon une expression utilisée par Vance R. Holloway ("La circunscripción del sujeto : metaficción, mito e identidad en la narrativa de José María Merino ", Anales de la Literatura Española Contemporánea, vol. 21, issues 1-2, 1986, p. 91) au sujet d'un phénomène similaire dans La orilla oscura.

22. Frank Wagner, "Du métatextuel à la métatextualité ", dans Alain Tassel (Textes réunis et présentés par), Narratologie. La métatextualité, Université de Nice-Sophia Antipolis, n 3, 2000, p. 17. En italique, cite Jean Ricardou, Pour une théorie du Nouveau Roman, Paris, Seuil, 1971, p. 32. 
El vuelo fue muy breve y Julio Lesmes pudo contemplar las islas, perdidas en la inmensidad oscura del mar, la mayor como un óvalo y la otra alargándose cerca de ella, como un pequeño hueso con voluminosas apófisis en los extremos. Ambas -con los islotes que las enlazaban, entre el reverbero amarillento que las hacia resplandecer bajo las nubes dispersas- presentaban la forma de uno de aquellos espejos de metal bruñido que servian para el acicalamiento de las damas en algún imperio antiguo. (p. 296)

Il va sans dire que le Patio dans lequel pénètre Madgalena brille de mille feux. Mais ce qu'il faut dire encore, c'est que, selon cette équivalence que José María Merino établit entre le territoire des rêves et celui de la littérature, et qui s'est confirmée très nettement dans ses dernières productions ${ }^{23}$, le statut de constructions imaginaires des romans dont on traite se trouve également être signifié par les marques d'onirisme dont est empreinte la narration de l'expérience des personnages, notamment lors de leur voyage ou séjour sur les îles. Tel est le biais, en particulier, par lequel Merino a cherché à conférer une dimension métalittéraire à El lugar sin culpa, comme il ressort de cette déclaration:

Quise también que en la novela no sólo se pudiesen conjugar lo estrictamente histórico con lo mítico, sino que hubiese un espacio abierto a la posibilidad de lo metaliterario, de modo que el lector pudiese sospechar, si quiere, que la verdadera huida de la doctora protagonista está en el espacio de la imaginación, de la ensoñación. Pero es algo que queda apuntado sólo lo suficiente ${ }^{24}$.

Indiquée par la somnolence ou la torpeur fréquente des personnages, mais aussi par le balancement de l'hélicoptère ou le mouvement hypnotique des vagues, l'ambiguïté entre expérience vigile et rêve persiste dans cette œuvre jusque dans le très beau final, caractérisé par un va-et-vient (ou une oscillation) spatial entre la maison de la protagoniste sur le continent (qu'elle n'a sans doute jamais quittée) et l'île où elle s'est réfugiée. Immobilisme physique peut-être, ainsi que le suggère encore la reprise de la première phrase du roman au début du dernier chapitre ("Hay una lagartija sobre el alféizar »), toujours est-il que ce voyage lui aura permis de réfléchir sur sa vie et de prendre des décisions propres à améliorer sa relation avec les siens.

Les effets de circularité relevant de la composition de ces récits, tel celui qui vient d'être évoqué ou encore celui créé par la rotation des chapitres à l'intérieur de chacune des parties de El centro del aire, s'inscrivent aussi parmi les procédés d'autoréférentialité en vigueur dans ces textes. Rappelant la forme d'une île, la circularité trouve encore sa traduction dans les volumes arrondis de l'habitat ou de la végétation sur l'île finale de El centro del aire, dans les images de tourbillons introduites dans El lugar sin culpa, ou encore dans la roue du

23. Notamment à travers les Cuentos del libro de la noche (Madrid, Alfaguara, 2005). On peut aussi noter que, une fois arrivé aux États-Unis, le protagoniste de El heredero fait une thèse sur le rêve dans l'œuvre de Galdós...

24. José María Merino, dans E. Gancedo, "José María Merino presentó en el Club de Prensa El lugar sin culpa ", Diario de León, 15/05/2007 [dernière consultation le 6 mai 2013]. Disponible sur : http : //www.diariodeleon.es/.../ 
destin qui pèse, thématiquement, sur El heredero (p. 397). Celui-ci présente, en outre, la particularité d'accueillir en son sein quelques séquences très autonomes semblables à des îlots textuels (par exemple, celle intitulée «UNA POESIA », p. 225-231), ce qui peut justifier la métaphore du collier de perles (p. 364), tout autant que la myriade d'îles dont il est question dans ce roman. Ainsi apparait-il, plus largement, que la circularité s'inscrit dans l'expression de l'autonomie des textes au regard du réel. Se donnant à voir et se définissant comme des microcosmes, les îles véritables ou métaphoriques de ces récits, avec leurs barrières de corail et leurs falaises, ont, en effet, pour vocation de signifier cette autonomie que José María Merino reconnaît depuis toujours à la littérature, voyant en elle non pas un monde subsidiaire au réel, mais parallèle à lui sans être pour autant complètement étanche, l'une de ses fonctions consistant à mettre en lumière, à sa façon, des aspects non perceptibles dans le chaos de la réalité factuelle : "A través de la literatura conocemos las cosas de una forma diferente, única. $"^{25}$ Soit une communication entre ces deux champs (fiction et réalité) signifiée, dans ces récits, par les liens établis, ou les ponts tendus (El heredero), entre l'île et le continent.

Mais là où l'on touche de plus près à l'hyper-littéralité, c'est lorsque Merino, par l'intermédiaire d'un poète fou dont il introduit la figure dans le dernier des romans dont on traite, donne à voir le mot comme une île " précieuse et véritable, se suffisant à elle-même, n'ayant besoin d'aucun ajout pour atteindre la pleine expressivité » (p. 44, je traduis). Et il ne nous échappe pas que prôner cette poésie dépouillée et absolue c'est, en quelque sorte, de la part de l'auteur de ces ouvrages, revendiquer le "réalisme " vers lequel il s'est acheminé au fil de quelque trente années consacrées à la création littéraire.

Au terme de cette étude, on arrive donc au constat, à propos des trois œuvres examinées, de la pratique par leur auteur d'une référentialité peu marquée, distanciée, comme absorbée par la fiction, ce qui me semble tout à fait en accord avec cette « victoire de l'imagination littéraire sur le référent naturel et factuel ${ }^{26}$ par laquelle, s'élevant contre ceux qui voudraient faire de ce dernier l'objet essentiel et même l'unique raison de la création romanesque, José María Merino identifie un «bon roman»:

Por un proceso de abstracción, las buenas novelas, capaces de vigencia temporal en un momento dado, acaban rebasando sus posibles referentes contemporáneos, y adquieren un carácter alegórico que las hace aún más decisivas e imprescindibles para conocer la realidad natural y fáctica con una profundidad que nunca permitiria la pura enumeración de los datos y los hechos desnudos ${ }^{27}$.

En ce sens, les récits de José María Merino sont loin de faire partie de ces "nouveaux espaces de la fiction " caractérisés par une injection massive de documents véridiques et de citations d'énoncés factuels, que Geneviève

25. José María Merino, dans "He querido contraponer... ", art. cit.

26. José María Merino, "Los parajes de la ficción ", art. cit., dans Ficción continua, op. cit.,p. 113.

27. Ibid.,p. 114. 
Champeau a repérés dans le paysage actuel du roman espagnol ${ }^{28}$. Pour leur part, sans renoncer à parler du réel - et loin de là -, les romans de José María Merino n'ont jamais cessé d'être des espaces de fiction, et de s'affirmer comme tels à travers les multiples procédés d'autoréférence dont on a parlé, certains d'eux, comme l'ambiguïté onirique, rappelant ceux utilisés par l'auteur du Quichotte.

\section{Bibliographie}

Bravo Frédéric, "L'analyse littérale », dans Nadine Ly (Textes réunis par), Littéralité 3. Limage dans le tapis, Bordeaux, Presses Universitaires de Bordeaux, Maison des Pays Ibériques, 1997, p. 43-74.

Champeau Geneviève (Textes réunis par), Référence et autoréférence dans le roman espagnol contemporain, Bordeaux, Maison des Pays Ibériques, 1994, 188 p.

— et alii (ed.), Nuevos derroteros de la narrativa española actual, Prensas Universitarias de Zaragoza, 2011, 397 p.

Dpto. prensa Alfaguara, "He querido contraponer la vida humana, racional, a la impasibilidad atemporal de la naturaleza ", interview de José María Merino éditée le 26/04/2007 [dernière consultation le 6 mai 2013]. Disponible sur : http : //www. alfaguara.com/.../

Eco Umberto, Lector in fabula. Le rôle du lecteur, Paris, Grasset, 1985, 314 p.

Gancedo E., "José María Merino presentó en el Club de Prensa El lugar sin culpa", Diario de León, 15/05/2007 [dernière consultation le 6 mai 2013]. Disponible sur : http : //www.diariodeleon.es/.../

García Carlos Javier, La invención del grupo leonés, Gijón, Júcar, 1994, 106 p.

Hamon Philippe, «Un discours contraint », dans Barthes R. et alii, Littérature et réalité, Paris, Seuil, 1982, p. 119-181.

Holloway Vance R., « La circunscripción del sujeto : metaficción, mito e identidad en la narrativa de José María Merino ", Anales de la Literatura Española Contemporánea, vol. 21, issues 1-2, 1986, p. 85-101.

Marín A. Diego, "Ahora se consume mucha pseudoliteratura, filones comerciales», Diario La Rioja, Cultura, 28/11/2006 [dernière consultation le 6 mai 2013]. Disponible sur: http://www.larioja.com/.../ahora-consume-muchapseudoliteratura_20061128.html

Merino José María, El centro del aire, Barcelona, Alfaguara Hispánica, 1991, 350 p.

— Días imaginarios, Barcelona, Seix Barral, 2002, 250 p.

- El heredero, Barcelona, Alfaguara, 2003, 402 p.

28. Geneviève Champeau et alii (ed.), Nuevos derroteros de la narrativa española actual, Prensas Universitarias de Zaragoza, 2011, p. 10. 
- Cuentos del libro de la noche, Madrid, Alfaguara, 2005, 167 p.

- El lugar sin culpa. Los espacios naturales, Barcelona, Alfaguara, 2007, 167 p.

- Ficción continua, Barcelona, Seix Barral, 2004, 380 p.

— "Reflexión sobre mi narrativa a la luz del Quijote ", dans Andrés-Suárez Irene, Casas Ana (ed.), Cuadernos de Narrativa, Neuchâtel, 1996, 1, p. 67-93.

— "Secretos de El heredero ", El heredero, dans Fernando Valls (edición introducción y notas de), Madrid, Barcelona, Clásicos Castalia, 2011, p. 77-80.

Noyaret Natalie, "José María Merino ", dans Natalie Noyaret (ed.), La narrativa española de hoy (2000-2010). La imagen en el texto (I), Bern, Peter Lang, 2011, p. $75-101$.

Riffaterre Michael, "L'illusion référentielle ", dans R. Barthes et alii, Littérature et réalité, op. cit., p. 91-118.

Valls Fernando (edición, introducción y notas), José María Merino. El heredero, Barcelona, Clásicos Castalia, col. «Narrativa siglo XXI», 2011, 510 p.

Wagner Frank, "Du métatextuel à la métatextualité », dans Alain Tassel (Textes réunis et présentés par), Narratologie. La métatextualité, Université de Nice-Sophia Antipolis, n 3, 2000, p. 13-28. 